\title{
Review
}

\section{Pathophysiology of Antihypertensive Therapy with Diuretics}

\author{
Michio FUKUDA ${ }^{1)}$ and Genjiro KIMURA ${ }^{1)}$
}

\begin{abstract}
Recent progress in antihypertensive therapy has widened the selection of drugs, and large clinical trials have attracted attention to newer classes of antihypertensives. Consequently, the use of diuretics as antihypertensive agents has been relatively reduced, particularly since the newer drugs are associated with fewer adverse metabolic reactions. However, diuretics have a specific activity of removing sodium from the body fluid, thereby rendering the blood pressure insensitive to sodium intake, relieving the overload to systemic circulation, and normalizing the circadian rhythm of blood pressure from a non-dipper to a dipper pattern. At low doses, diuretics are known to be as effective as all other antihypertensive agents for reducing nearly all types of cardiovascular events. In this brief review, the indication for thiazide diuretics will be discussed based on the pathophysiology of hypertension and antihypertensive therapy with diuretics mainly from the point of view of sodium metabolism. Low-dose diuretics will continue to be an important agent in the treatment of hypertension, mostly in combination with vasodilators such as modulators of the reninangiotensin system and calcium channel blockers. (Hypertens Res 2006; 29: 645-653)
\end{abstract}

Key Words: diuretics, diuretic resistance, pressure-natriuresis, circadian rhythm, combination therapy

\section{Introduction}

Diuretics, particularly thiazides and their analogs, exert a gradual and stable hypotensive effect when taken once daily. They also have many other benefits, including few absolute contraindications, good compliance and low cost. Therefore, they have long been widely used as antihypertensive drugs. In recent large-scale clinical studies, diuretics have been shown to prevent cardiovascular events, particularly cerebrovascular disorders $(1,2)$. At low doses, diuretics are as effective as all other antihypertensive agents for nearly all types of cardiovascular events (2). Based on these evidences, the 7th Report of the U.S. Joint National Committee on Prevention, Detection, Evaluation and Treatment of Blood Pressure (JNC 7) recommended that thiazide diuretics be used preferentially as the antihypertensive drugs of choice $(3,4)$. The report also recommended that when two or more antihypertensive agents are combined, one of the drugs should be a thiazide diuretic $(3,4)$. On the other hand, the range of selected antihypertensives has been expanded, because agents with different mechanisms of action have been developed, including calcium channel blockers and renin-angiotensin (RA) modulators such as angiotensin-converting enzyme (ACE) inhibitors and angiotensin receptor blockers (ARB). Consequently, the use of diuretics has greatly decreased due to concern over adverse metabolic reactions. There is therefore increasing momentum for reexamining the proper use of diuretics. This article is an attempt to elucidate the characteristics of hypertensive patients for whom a diuretic is indicated and to clarify the pathophysiological basis for selecting these drugs.

From the ${ }^{1}$ Department of Internal Medicine and Pathophysiology, Nagoya City University Graduate School of Medical Sciences, Nagoya, Japan.

This study was supported by Research Grants for Cardiovascular Diseases (C-2001-5) from the Ministry of Health and Welfare of Japan, as well as Grants from Nagoya City University, the Salt Science Research Foundation (No. 04C1), Metabolic Disorders Treatment Research Foundation, Aichi Kidney Foundation and Japan Cardiovascular Research Foundation, and a Grant-in-Aid for Scientific Research (C) from the Ministry of Education, Culture, Sports, Science and Technology of Japan through the Japan Society for the Promotion of Science.

Address for Reprints: Genjiro Kimura, M.D., Department of Internal Medicine and Pathophysiology, Nagoya City University Graduate School of Medical Sciences, Mizuho-cho, Mizuho-ku, Nagoya 467-8601, Japan. E-mail: genki@med.nagoya-cu.ac.jp

Received April 3, 2006; Accepted in revised form June 18, 2006. 


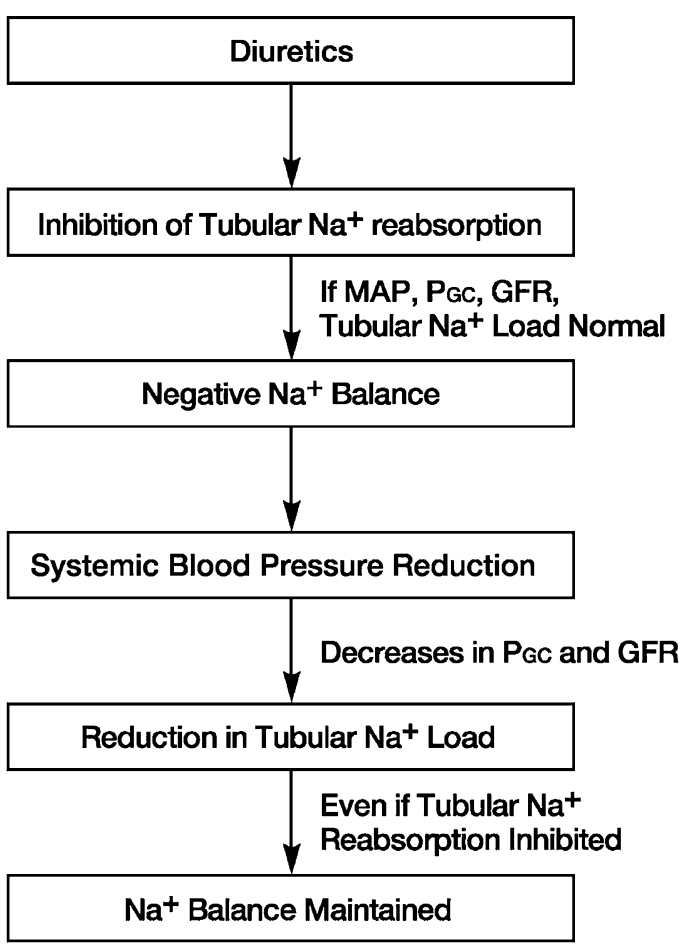

Fig. 1. The antihypertensive mechanism of diuretics. Diuretics inhibit tubular sodium reabsorption and increase the slope of the pressure-natriuresis curve (see Fig. 2) by multiplying urinary sodium excretion per unit change in glomerular filtration rate (GFR) and renal tubular sodium load, i.e., per unit change in systemic mean arterial pressure (MAP) and glomerular capillary pressure $\left(\mathrm{P}_{G C}\right)$.

\section{Mechanisms of the Hypotensive Action of Diuretics}

Fundamental to the action of thiazide diuretics is the enhancement of urinary sodium excretion through the inhibition of $\mathrm{Na}-\mathrm{Cl}$ co-transport in the distal renal tubules. The precise mechanisms by which the inhibition of renal sodium reabsorption lowers blood pressure (BP) remain unknown. We have proposed that one of the following conditions is necessary to elevate BP and cause hypertension: 1) an increase in vascular resistance between the heart and the glomeruli; 2) a decrease in the glomerular filtration capability; or 3) an enhancement in renal sodium reabsorption by tubules (5). The first of these three conditions creates sodium-insensitive hypertension, while the latter two create sodium-sensitive hypertension (5). Considering the opposite of case 3), of itself, is useful for elucidating the hypotensive mechanism of diuretics (Fig. 1) (6-8). If the glomerular filtration rate (GFR) and the tubular sodium load are normal, inhibition of the tubular sodium reabsorption by a diuretic makes the sodium balance negative. Consequently, both the body fluid volume

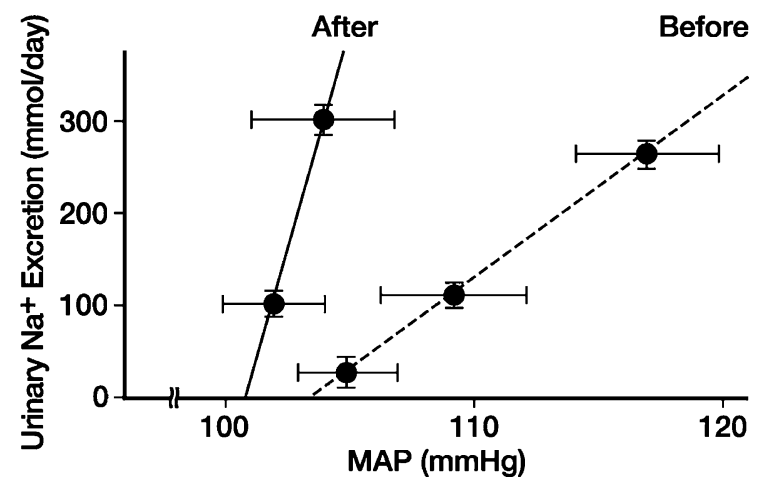

Fig. 2. The effect of diuretics on the pressure-natriuresis curve. The pressure-natriuresis curves were compared before (dotted line) and after (solid line) administration of a diuretic, mefruside. The diuretic increased the slope, but did not affect the blood-pressure axis (from Saito and Kimura (6)).

and BP decrease. A reduction in glomerular capillary pressure accompanying the reduction in systemic BP results in decreases in GFR and tubular sodium load, thereby maintaining sodium balance against the inhibited sodium reabsorption. Thus, it is understood that an inhibition of tubular sodium reabsorption as occurs with the use of a diuretic necessitates reductions in BP and essentially in tubular sodium load to maintain sodium balance (6-8). In fact, a recent report demonstrated a reduction in glomerular capillary pressure with diuretics (9).

Diuretics frequently induce an elevation of serum creatinine. This reflects inevitable functional changes necessitated by decreases in GFR, glomerular capillary pressure and tubular sodium load with diuretic treatment, as discussed above. In the recent ALLHAT $(1)$ and INSIGHT $(10,11)$ studies, decreased GFR was confirmed in the groups treated with diuretics. Reflecting a reduction in glomerular capillary pressure, however, elevated serum creatinine suggests a renoprotective effect, which was of concern a decade ago, when RA modulators were in wide use (12). In fact, a reduction in urinary protein equal to that seen with RA modulators was recently demonstrated with diuretics $(13,14)$. This is a noteworthy point that must be examined in the future by largescale clinical studies.

\section{Effect of Diuretics on the Pressure-Natriuresis Curve}

The relationship between sodium balance and BP, mentioned above, is expressed well by Guyton's pressure-natriuresis curve (15-17). We examined the hypotensive effect of the thiazide diuretic, mefruside, based on this curve (Fig. 2) (6). Mefruside increased the slope of the pressure-natriuresis curve, but did not result in a significant change in the inter- 

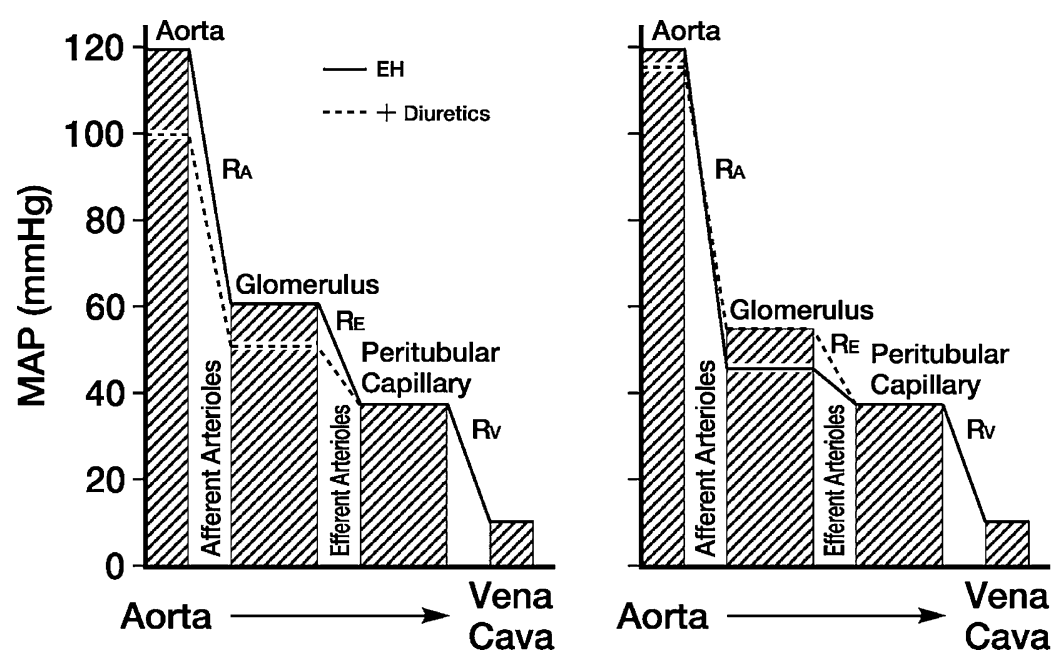

Fig. 3. Estimated changes in glomerular hemodynamics with diuretic administration. The changes in glomerular hemodynamics with diuretics were compared between responders for whom a favorable hypotensive effect was obtained (left) and resistant patients for whom this effect was not obtained (right). Note that vascular resistance in the afferent arterioles varies greatly between the two groups. In resistant patients, reduction in vascular resistance at this site is essential to reduce systemic blood pressure, suggesting the importance of concomitant therapy with vasodilators. EH, essential hypertension; MAP, mean arterial pressure; $\mathrm{R}_{A}$, afferent arteriolar resistance; $\mathrm{R}_{E}$, efferent arteriolar resistance; $\mathrm{R}_{V}$, renal vein resistance.

cept of the BP axis. This finding suggests that the hypotensive effect of diuretics is based mainly on the increase in the slope of the curve by multiplying the rate of sodium excretion per unit change in BP. Therefore, diuretic treatment enables the maintenance of sodium balance at lower levels of BP, GFR and tubular sodium load (6-8). Among life-style modifications, the Dietary Approaches to Stop Hypertension (DASH) diet, which is rich in vegetables, fruits, and low-fat dairy products, has drawn attention as one of the approaches with the greatest hypotensive effect (18-20). The effectiveness of the DASH diet is based on its diuretic action (8), and the diet thus appears to be a natural diuretic (8).

The reciprocal of the slope of the pressure-natriuresis curve represents the change in BP when sodium intake is modified. In other words, it represents the sodium sensitivity of BP $(5$, 21). Therefore, we can also imagine that diuretics exert their hypotensive effect by reducing sodium sensitivity, as reflected in the fact that a stronger hypotensive effect is recognized in patients with a more depressed slope of the pressure-natriuresis curve and higher sodium sensitivity $(6,8,20)$.

\section{Mechanism of Resistance to Diuretics}

We next turn to the question of why there are differences in hypotensive effect among patients in whom a similar diuretic action is seen, and will examine the phenomenon whereby no significant hypotensive effect is seen despite the occurrence of a diuretic action, i.e., the mechanism of diuretic resistance.

To maintain sodium balance with inhibited tubular sodium reabsorption under diuretic administration, the tubular sodium load must be reduced. Specifically, glomerular capil- lary pressure and GFR should be reduced. If BP does not decrease in this case, a large pressure difference must be developed between the systemic circulation and glomerular capillaries. An increase in vascular resistance between the heart and glomeruli, mainly in the afferent arterioles, as a result of diuretic administration prevents manifestation of the hypotensive effect of the drug (Fig. 3).

Although the precise mechanism of diuretic resistance due to the increase in afferent arteriolar resistance is not clear, it has been speculated that stimulation of the sympathetic nerve and RA systems as well as tubulo-glomerular feedback are involved. For example, thiazide diuretics, which promote natriuresis downstream from the macula densa, have a strong hypotensive effect despite their weak diuretic action. On the other hand, loop diuretics, which act before reaching the macula densa, have a weak hypotensive effect despite their potent diuretic action, because they inhibit $\mathrm{NaCl}$ transport in the macula densa and thereby stimulate the RA system. Under conditions where these factors are strongly in effect, the pressure-natriuresis curve is usually shifted rightward without depressing the slope. A steep slope is characteristic of sodium insensitivity of $\mathrm{BP}(5,21,22)$. As mentioned previously, the shallower the slope of the pretreatment pressure-natriuresis curve and the greater the sodium sensitivity of BP, the greater the improvement in the slope and the greater the hypotensive effect of diuretic therapy $(6,8)$. Conversely, patients with a pressure-natriuresis curve with a steeper slope and sodium insensitivity are resistant to diuretics in addition to sodium intake restriction $(6,8,22)$. The factors suggestive of diuretic resistance are summarized in Table 1. 
Table 1. Factors That Cause Diuretic Resistance

\begin{tabular}{l} 
Factors \\
\hline Sodium insensitivity \\
Increased activities in RA and sympathetic nerve systems \\
Increase in tubulo-glomerular feedback \\
Use of loop diuretics \\
Nonsteroidal anti-inflammatory drugs \\
Cyclosporins \\
Renovascular hypertension
\end{tabular}

All of these, listed in this table, are factors that increase vascular resistance between the heart and glomeruli, at the same time resulting in sodium-insensitive hypertension. Conversely, factors that increase sodium sensitivity of blood pressure are advanced age, black ethnicity, female sex, obesity, and severe hypertension. Concomitant nephropathy clearly increases sodium sensitivity. Increased sodium sensitivity strengthens the hypotensive effect of diuretics.

\section{Indications for Diuretic Therapy Based on the Pressure-Natriuresis Curve}

During early onset of essential hypertension, the slope of the pressure-natriuresis curve is generally normal and steep with a slight rightward shift in parallel (Fig. 4) (23-25). In other words, the patients are sodium-insensitive. By contrast, as hypertension progresses from moderate to severe, the pressure-natriuresis curve is gradually shifted further rightward and its slope gradually decreases, resulting in higher sodium sensitivity of BP (23-25).

Taken together, this natural history of the pressure-natriuresis curve for essential hypertension and the above-mentioned effect of diuretics on the pressure-natriuresis curve clearly show the indication of diuretics on hypertension $(6,26)$. In borderline or mild essential hypertension, the pressure-natriuresis curve is shifted rightward but the slope remains steep, indicating sodium insensitivity. Therefore, no significant hypotensive effect can be expected with diuretic therapy alone in these cases. In fact, in an investigation of the efficacy of monotherapy with various antihypertensive drugs in white patients below 60 years old, who are assumed to have a steep slope of the curve and therefore to be sodium insensitive, the efficacy rate for thiazide diuretics was around $30 \%$, the lowest of the drug classes examined $(27,28)$. In black patients older than 60 years, for whom the slope of the curve is assumed to be depressed and to be sodium sensitive, the highest efficacy rates were seen for calcium channel blockers and diuretics $(27,29)$. The cardiovascular protection by treatment with thiazide diuretics in elderly hypertensive patients has been demonstrated in large clinical studies such as the SHEP study $(30-32)$. It is therefore easy to understand why international guidelines consider hypertension in elderly patients to be a positive indication for diuretic treatment $(3,4,33-35)$.

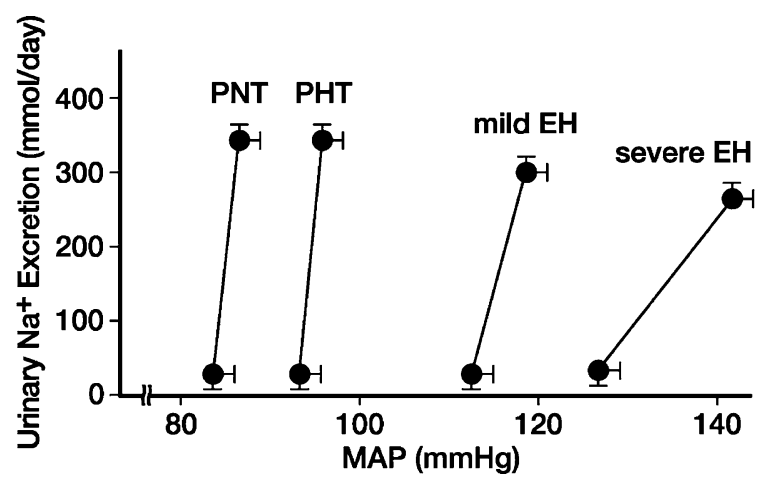

Fig. 4. Natural history of the pressure-natriuresis curve in essential hypertension. Although the slopes of the pressurenatriuresis curves of children whose parents are both normotensive (PNT) and those whose parents are both hypertensive (PHT) are identically steep, the curves of the latter are shifted rightward. This finding suggests that renal afferent arteriolar resistance increases before the onset of hypertension in individuals predisposed to hypertension. The slopes are steep during the early stages of essential hypertension, with sodium insensitivity. As the condition progresses to mild essential hypertension (mild EH) and severe essential hypertension (severe EH), the curves shift further rightward. At the same time, their slopes become depressed and shallower, with an observed shift toward sodium-sensitive hypertension (from Parfrey (23)).

\section{Diuretics Normalize Circadian Rhythm of Blood Pressure}

In a study by Uzu and Kimura, the circadian BP rhythm was examined before and after administration of the thiazide diuretic, hydrochlorothiazide, once each morning for 4 weeks (36). With the focus on the reduction in BP from daytime to nighttime, a significant reduction in nocturnal BP was seen in both the dipper and non-dipper groups. However, an interaction between the diuretic and the reduction in nocturnal BP was present only in the non-dipper group; no interaction was seen in the dipper group. These findings clearly show that the diuretic did not affect the reduction in nocturnal BP in dippers, but it enhanced the reduction in nocturnal BP in nondippers, shifting their circadian BP rhythm toward the dipper pattern (Fig. 5) (36).

The non-dipper pattern of circadian BP rhythm, in which the reduction in nocturnal $\mathrm{BP}$ is impaired, is observed not only with essential hypertension (36-39), but also with primary aldosteronism (40), hypertension in blacks $(41,42)$, chronic kidney disease (43), and diabetic nephropathy (38), all of which are characterized by diminished renal functional reserve and high sodium sensitivity (44). The same shift from non-dipper to dipper status, as recognized by dietary sodium intake restriction (38), has also been confirmed with diuretics 

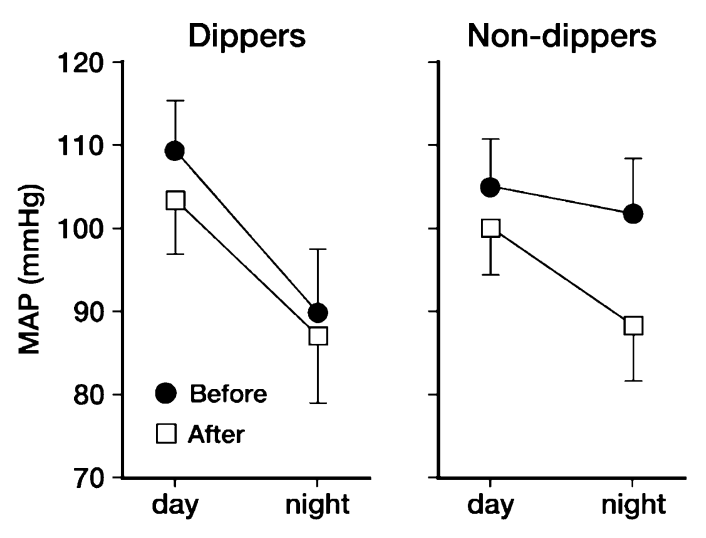

Fig. 5. Normalization of circadian blood pressure (BP) rhythm by diuretics. Effects of a thiazide diuretic, hydrochlorothiazide, on circadian BP rhythm were compared between dippers (left) and non-dippers (right). Dippers showed a significant reduction in nocturnal BP independently of administration of the diuretic. In contrast, in non-dippers, the previously impaired reduction in nocturnal BP was restored by the diuretic. Moreover, a significant interaction was seen between the reduction in nocturnal BP and diuretic administration $(\mathrm{p}<0.001)$, with the reduction being enhanced by the diuretic. Thus, the diuretic clearly shifted the circadian BP rhythm from the non-dipper type to the normal dipper type. $M A P$, mean arterial pressure (from Uzu and Kimura (36)).

(36), suggesting that the kidney's ability to excrete sodium plays an important role in determining the circadian BP rhythm $(43,44)$.

A high risk of cardiovascular complications has repeatedly been reported for patients with hypertension who exhibit a non-dipper circadian BP rhythm (45-49). This is consistent with the idea that nocturnal BP is more important than diurnal $\mathrm{BP}$ or 24-h average BP as a determining factor of cardiovascular events (50-52). Recently, renal dysfunction has drawn attention as a potent risk factor or predictor for cardiovascular events (53-57). Progression from microalbuminuria to albuminuria, renal dysfunction, and eventual end-stage renal failure as a function of renal functional loss is associated with a pronounced increase in cardiovascular events (58). Moreover, these risk factors are independent of classical risk factors such as diabetes and hypertension. We previously found that the greater the decrease in GFR with chronic kidney disease, the greater the elevation in nocturnal $\mathrm{BP}$, and that nocturnal urinary sodium and protein excretions increased at the same time (43). In kidney transplantation donors, nocturnal BP increased in proportion to the decrease in GFR following unilateral nephrectomy (59). These findings have prompted speculation that a decrease in renal function prevents waste products and sodium from being completely excreted during the daytime and that BP reduction at night must be suppressed in order to vigorously drive nighttime renal function, which results in the non-dipper pattern $(44,60)$. We propose that this non-dipper circadian BP rhythm would give rise to cardiovascular events as a function of renal functional loss $(60)$. This hypothesis is consistent with reports that the non-dipper pattern was observed in patients with sodium-sensitive hypertension $(36,38,44)$, who are at high risk for cardiovascular events (61-63).

The fact that, like sodium intake restriction (38), diuretics normalize the circadian BP rhythm from a non-dipper to a dipper-type pattern (36) must be noted as one of the potent mechanisms by which diuretics reduce cardiovascular events.

\section{Low-Dose Diuretics and Their Significance in Combination Therapies}

Based on these findings, it seems clear that resistance to the hypotensive effect of diuretics is caused not by a weakening of the diuretic response to these drugs, but rather by excessive constriction of the afferent arterioles. Therefore, diuretic resistance should be handled not by rashly increasing the dose of the diuretic, but rather by switching to another drug or administering the diuretic in combination with the other drugs. In fact, it has been shown that reducing the dose to $1 / 8$ of the conventional dose results in little change in the hypotensive effect $(64,65)$. It should be noted that a nearly full hypotensive effect of diuretics is obtained with a low dose; thus, the effect is not dose-dependent (64).

Patients with sodium sensitivity, who are thought to achieve a favorable effect from diuretic therapy, are generally older and severely hypertensive, and they often have decreased cardiac output and organ blood flow. It is therefore recommended that the lowest effective diuretic dose be used and that the diuretic be administered in combination with a calcium channel blocker or RA modulator that has a vasodilatory effect $(6,25,26)$. In keeping with the rationale described above (see Fig. 3), these vasodilatory agents will likely release afferent arteriolar vasoconstriction and potentiate the hypotensive effect of the diuretic. This will be discussed in detail in a later section describing the algorithm for concomitant use (below).

Diuretics such as thiazides are known to dose-dependently elicit adverse reactions such as hypokalemia, hypomagnesemia, hyperuricemia, hyperlipidemia and glucose intolerance (64). Antihypertensive therapy with thiazide diuretics also has been shown to increase the risk of sudden death in a dose-dependent fashion (65). It has been suggested that to reduce this risk, either the thiazide dose must be reduced or a potassium-sparing diuretic must be concomitantly administered (65). Caution should be exercised regarding reports indicating the absence of an inhibition of cardiovascular events in cases where hypokalemia occurs even at a low dose (66). Recently, there have been reports suggesting that while the likelihood of new-onset diabetes mellitus clearly increases with diuretic use, this increase is not necessarily linked to cardiovascular events or death $(67,68)$. 

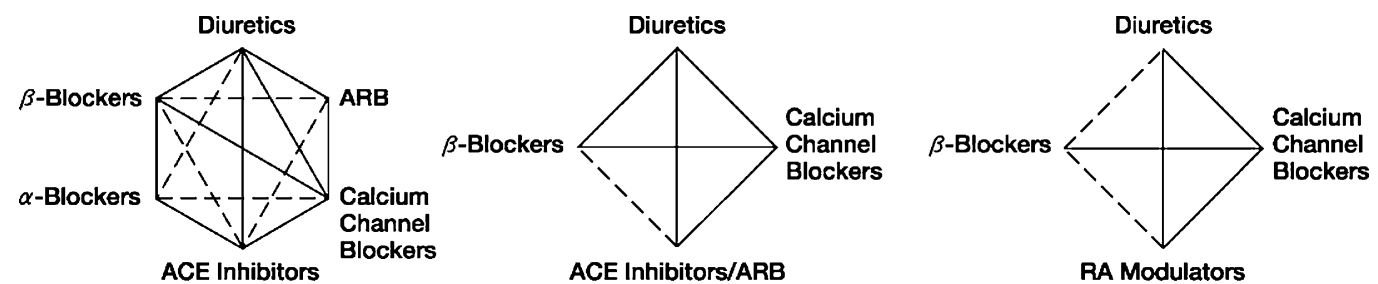

Fig. 6. Algorithm for concomitant diuretic use. The ESH/ESC 2003 algorithm (left) for concomitant use of antihypertensives was modified. In step 1 (center), antihypertensive agents were limited to those frequently prescribed as first-line drugs in routine clinical settings. ACE inhibitors and ARBs were combined as RA modulators. In step 2 (right), the algorithm was modified based on the recent findings for concomitant use of a diuretic and a $\beta$-blocker, which could have a synergistic negative effect on both organ blood flow and glucose metabolism, which is undesirable.

In any case, low-dose diuretic therapy confers an adequate hypotensive effect, with only adverse reactions increasing dose-dependently $(66,69)$.

Thus, it is recommended that antihypertensive diuretics such as the thiazides be administered in combination with various other antihypertensive drugs rather than alone, and that they be administered at a low dose.

\section{New Algorithm for Concomitant Administration}

The best known algorithm for determining desirable combinations of antihypertensive drugs is that proposed in the 2003 ESH/ESC Guidelines (33). We simplified this algorithm to create a more practical model (Fig. 6). First, $\alpha$-blockers are eliminated, because they are almost never used as first-line agents $(1,3,4)$. Keep in mind, however, that $\beta$-blockers are the only drugs desirable for concomitant use with $\alpha$-blockers. Next, attention is focused on the fact that concomitant use of ACE inhibitors and ARBs is undesirable from the perspective of antihypertensive efficacy (70). Moreover, the method for combining an ACE inhibitor or ARB with a desirable concomitant drug is the same. Therefore, if these two types of drugs are combined as RA modulators, the algorithm can be simplified as indicated in the center of Fig. 6. In addition, the need arose to modify the algorithm for concomitant use of $\beta$ blockers and diuretics. In the LIFE study, although equal reductions in BP were seen in the ARB group and the $\beta$ blocker group, the inhibition of cardiovascular events in the former was significantly better than that in the latter $(71,72)$. A subsequent repeat analysis of this study showed that the rate of concomitant diuretic use was approximately $70 \%$ in both groups (73). $\beta$-Blockers and diuretics both have pharmacologic effects that reduce cardiac output (26). This suggests that concomitant use of these drugs may have increased the rate of cardiovascular events such as stroke $(71,72)$, and probably cerebral infarction, due to a decrease in organ blood flow. The combination of diuretics and ARBs, the latter of which increase cardiac output, appears to bring out the strengths of each type of drug (26). The recently published ASCOT-BPLA study can be interpreted in the same manner
(74). In this study, as compared with concomitant administration of a $\beta$-blocker and a diuretic, the combination of a calcium channel blocker and an ACE inhibitor significantly inhibited cardiovascular events. The fact that there was a difference in stroke occurrence that could not be explained by correcting for factors such as BP (75) suggests that organ blood flow may have decreased with the former combination. With concomitant administration of diuretics and $\beta$-blockers, there is also concern that abnormal glucose metabolism may be exacerbated $(34,68,71,74)$. Based on these evidences, it was determined that combining diuretics and $\beta$-blockers is undesirable, and the algorithm was modified accordingly. The final form of the algorithm is shown in the right side of Fig. 6.

Examining this algorithm with a focus on diuretics serves as a reminder that the drugs for concomitant use are limited to RA modulators and calcium channel blockers. Because both types of drugs are vasodilators, they can both maintain organ blood flow while potentiating the hypotensive effect of the diuretic by releasing afferent arteriolar vasoconstriction, the key to inducing diuretic resistance. Rates of concomitant diuretic administration were examined in clinical trials provided in the JNC 7 guidelines in the U.S. as organ protection by RA modulators $(3,4)$. It is of particular interest that, for both ACE inhibitors and ARBs, diuretics were used as concomitant drugs in nearly all of the studies on heart failure, chronic kidney disease and stroke. These findings may suggest that concomitant use of diuretics and RA modulators, rather than RA modulators alone achieved a protective effect. It is assumed that the combination of the two types of drugs is advantageous in that each drug counteracts the adverse reactions of the other and that they synergistically potentiate their hypotensive effects $(6,8,25,26,76)$. It would also be of interest to examine whether these kinds of advantages exist with concomitant use of calcium channel blockers and diuretics. Regarding therapy focused on modulators of the RA system, large comparative clinical studies examining whether concomitant diuretic use is beneficial, as in the LIFE study ( 71 , 72 ), and whether concomitant calcium channel blocker use is beneficial, as in the ASCOT study $(74,75)$, are anticipated in the future. 


\section{Changes over Time in the Quantity of Diuretics Prescribed}

Sales of diuretics in the U.S., although once on the decline, have been increasing since 1996, and diuretics are now the most frequently prescribed hypotensive agents in the U.S. (77). It has been speculated that this reversal reflects the recognition of their ability, in lower doses, to provide excellent organ protection, hypotensive efficacy and reduced adverse reactions, as well as their economic advantages.

In contrast, sales of diuretic agents in Japan have remained nearly flat at a minimum level over the last 10 years, while those of calcium channel blockers have continued to increase, and those of ARBs have recently increased rapidly. A possible reason for the decrease in diuretic use in Japan is that the advantages of low-dose diuretic therapy have not yet been experienced in this country. The hypotensive effect of diuretics peaks at a low dose and thus is not dose-dependent, while adverse reactions increase in a dose-dependent fashion (64). In Japan, experience with diuretics has been limited to highdosages. It is therefore likely that the adverse reactions have been the most prominent aspect of diuretic therapy, resulting in a poor assessment of hypotensive diuretics. However, sodium intake in Japan is among the highest in the world, and sodium sensitivity of BP is presumed to be high in the Japanese. Therefore, it will be particularly important to carefully reexamine the proper use of diuretics in Japanese. It will also be important to vigorously promote the adoption of the DASH diet $(8,18,19)$, a natural diuretic that does not elicit hypokalemia or any other adverse reactions and that is healthy for all individuals. In any event, the era of rashly avoiding diuretic use appears to have ended.

\section{Perspectives}

There are a variety of views on how to select an appropriate agent when initiating hypotensive drug therapy. These are based on perspectives such as organ protection, a metabolic viewpoint that neither new onset of diabetes mellitus nor atherosclerosis is promoted and the need to avoid certain drugs, depending on the underlying disease or organ damage. We previously reported that the sodium sensitivity of BP occurring with diminished renal functional reserve reflects a predisposition to cardiovascular complications $(61,62)$. Diabetes mellitus and nephropathy increase sodium sensitivity. Decreasing the sodium load in sodium-sensitive patients with diuretics not only greatly reduces systemic BP but also normalizes the circadian BP rhythm, thus shifting from a nondipper to a dipper pattern. It is also expected to reduce other types of loads, such as glomerular hypertension $(14,36)$.

Thus, it appears that diuretics are essential for the treatment of a variety of pathophysiological conditions, and that the key to using these drugs is administration at low doses in combination with other drugs. If used properly, by taking into account the pathophysiology of the individual, diuretics will continue to play an important role in the treatment of hypertension.

\section{References}

1. ALLHAT Officers and Coordinators for the ALLHAT Collaborative Research Group, The Antihypertensive and Lipid-Lowering Treatment to Prevent Heart Attack Trial: Major outcomes in moderately hypercholesterolemic, hypertensive patients randomized to pravastatin $v s$ usual care: the Antihypertensive and Lipid-Lowering Treatment to Prevent Heart Attack Trial (ALLHAT-LLT). JAMA 2002; 288: 2998-3007.

2. Psaty BM, Lumley T, Furberg CD, et al: Health outcomes associated with various antihypertensive therapies used as first-line agents: a network meta-analysis. JAMA 2003; 289: 2534-2544.

3. Chobanian AV, Bakris GL, Black HR, et al: The Seventh Report of the Joint National Committee on Prevention, Detection, Evaluation, and Treatment of High Blood Pressure: the JNC 7 report. JAMA 2003; 289: 2560-2572.

4. Chobanian AV, Bakris GL, Black HR, et al: Seventh report of the Joint National Committee on Prevention, Detection, Evaluation, and Treatment of High Blood Pressure. Hypertension 2003; 42: 1206-1252.

5. Kimura G, Brenner BM: The renal basis for salt sensitivity in hypertension, in Laragh JH, Brenner BM (eds): Hypertension: Pathophysiology, Diagnosis and Management. New York, Raven Press, 1995, pp 1569-1588.

6. Saito F, Kimura G: Antihypertensive mechanism of diuretics based on pressure-natriuresis relationship. Hypertension 1996; 27: 914-918.

7. Kimura G, Uzu T, Nakamura S, Inenaga T, Fujii T: High sodium sensitivity and glomerular hypertension/hyperfiltration in primary aldosteronism. J Hypertens 1996; 14: 1463 1468 .

8. Akita S, Sacks FM, Svetkey LP, Conlin PR, Kimura G: Effects of the Dietary Approaches to Stop Hypertension (DASH) diet on the pressure-natriuresis relationship. Hypertension 2003; 42: 8-13.

9. Zhou X, Matavelli LC, Ono H, Frohlich ED: Superiority of combination of thiazide with angiotensin-converting enzyme inhibitor or AT1-receptor blocker over thiazide alone on renoprotection in L-NAME/SHR. Am J Physiol Renal Physiol 2005; 289: F871-F879.

10. Brown MJ, Palmer CR, Castaigne A, et al: Morbidity and mortality in patients randomised to double-blind treatment with a long-acting calcium-channel blocker or diuretic in the International Nifedipine GITS study: Intervention as a Goal in Hypertension Treatment (INSIGHT). Lancet 2000; 356: 366-372.

11. de Leeuw PW, Ruilope LM, Palmer CR, et al: Clinical significance of renal function in hypertensive patients at high risk: results from the INSIGHT trial. Arch Intern Med 2004; 164: 2459-2464.

12. Fukuda M, Kimura G: Can calcium channel blockers preserve renal function better than diuretics during antihypertensive treatment? Arch Intern Med 2005; 165: 1312.

13. Marre M, Puig JG, Kokot F, et al: Equivalence of indapam- 
ide SR and enalapril on microalbuminuria reduction in hypertensive patients with type 2 diabetes: the NESTOR Study. J Hypertens 2004; 22: 1613-1622.

14. Uzu T, Harada $\mathrm{T}$, Namba $\mathrm{T}$, et al: Thiazide diuretics enhance nocturnal blood pressure fall and reduce proteinuria in immunoglobulin A nephropathy treated with angiotensin II modulators. J Hypertens 2005; 23: 861-865.

15. Guyton AC: Arterial Pressure and Hypertension, Circulatory Physiology III. Philadelphia, WB Saunders, 1980.

16. Guyton AC: Renal function curve - a key to understanding the pathogenesis of hypertension. Hypertension 1987; 10: $1-6$.

17. Guyton AC: Dominant role of the kidneys and accessory role of whole-body autoregulation in the pathogenesis of hypertension. Am J Hypertens 1989; 2: 575-585.

18. Appel LJ, Moore TJ, Obarzanek E, et al, DASH Collaborative Research Group: A clinical trial of the effects of dietary patterns on blood pressure. N Engl J Med 1997; 336: $1117-$ 1124.

19. Sacks FM, Svetkey LP, Vollmer WM, et al: Effects on blood pressure of reduced dietary sodium and the dietary approaches to stop hypertension (DASH) diet. $N$ Engl J Med 2001; 344: 3-10.

20. Vollmer WM, Sacks FM, Ard J, et al: Effects of diet and sodium intake on blood pressure: subgroup analysis of the DASH-sodium trial. Ann Intern Med 2001; 135: 10191028.

21. Kimura G, Brenner BM: Implications of linear pressurenatriuresis relationship and importance of sodium sensitivity in hypertension. J Hypertens 1997; 15: 1055-1061.

22. Kimura G, Saito F, Kojima S, et al: Renal function curve in patients with secondary forms of hypertension. Hypertension 1987; 10: 11-15.

23. Parfrey PS: Salt in essential hypertension, in Sleight $P$, Freis ED (eds): Hypertension. Butterworth's International Medical Reviews, Cardiology 1. London, Butterworth, 1982, pp 322-339.

24. Parfrey PS, Markandu ND, Roulstone JE, Jones BE, Johnes JC, MacGregor GA: Relation between arterial pressure, dietary sodium intake and renin system in essential hypertension. Br Med J 1981; 283: 94-97.

25. Kimura G, Deguchi F, Kojima S, et al: Antihypertensive drugs and sodium restriction. Analysis of their interaction based on pressure-natriuresis relationship. Am J Hypertens 1988; 1: 372-379.

26. Kimura G, Abe H, Ashida T, et al: Hypotensive mechanisms of antihypertensives based on the water tank model. Am J Hypertens 1989; 2: 532-536.

27. Materson BJ, Reda DJ, Cushman WC, et al, The Department of Veterans Affairs Cooperative Study Group on Antihypertensive Agents: Single-drug therapy for hypertension in men. A comparison of six antihypertensive agents with placebo. N Engl J Med 1993; 328: 914-921.

28. Deary AJ, Schumann AL, Murfet H, Haydock SF, Foo RS, Brown MJ: Double-blind, placebo-controlled crossover comparison of five classes of antihypertensive drugs. $J$ Hypertens 2002; 20: 771-777.

29. Morgan T, Lauri J, Bertram D, Anderson A: Effect of different antihypertensive drug classes on central aortic pressure. Am J Hypertens 2004; 17: 118-123.
30. SHEP Cooperative Research Group: Prevention of stroke by antihypertensive drug treatment in older persons with isolated systolic hypertension. Final results of the Systolic Hypertension in the Elderly Program (SHEP). JAMA 1991; 265: 3255-3264.

31. MacMahon S, Rodgers A: The effects of blood pressure reduction in older patients: an overview of five randomized controlled trials in elderly hypertensives. Clin Exp Hypertens 1993; 15: 967-978.

32. Hebert PR, Moser M, Mayer J, Glynn RJ, Hennekens CH: Recent evidence on drug therapy of mild to moderate hypertension and decreased risk of coronary heart disease. Arch Intern Med 1993; 153: 578-581.

33. 2003 European Society of Hypertension-European Society of Cardiology guidelines for the management of arterial hypertension. J Hypertens 2003; 21: 1011-1053.

34. Williams B, Poulter NR, Brown MJ, et al: Guidelines for management of hypertension: report of the fourth working party of the British Hypertension Society, 2004-BHS IV. J Hum Hypertens 2004; 18: 139-185.

35. Japanese Society of Hypertension Guidelines Subcommittee for the Management of Hypertension: Guidelines for the management of hypertension for general practitioners. Hypertens Res 2001; 24: 613-634.

36. Uzu T, Kimura G: Diuretics shift circadian rhythm of blood pressure from nondipper to dipper in essential hypertension. Circulation 1999; 100: 1635-1638.

37. Uzu T, Kazembe FS, Ishikawa K, Nakamura S, Inenaga T, Kimura G: High sodium sensitivity implicates nocturnal hypertension in essential hypertension. Hypertension 1996; 28: $139-142$.

38. Uzu T, Ishikawa $\mathrm{K}$, Fujii $\mathrm{T}$, Nakamura $\mathrm{S}$, Inenaga $\mathrm{T}$, Kimura G: Sodium restriction shifts circadian rhythm of blood pressure from nondipper to dipper in essential hypertension. Circulation 1997; 96: 1859-1862.

39. Fujii T, Uzu T, Nishimura M, et al: Circadian rhythm of natriuresis is disturbed in non-dipper type of essential hypertension. Am J Kidney Dis 1999; 33: 29-35.

40. Uzu T, Nishimura M, Fujii T, et al: Changes in the circadian rhythm of blood pressure in primary aldosteronism in response to dietary sodium restriction and adrenalectomy. $J$ Hypertens 1998; 16: 1745-1748.

41. Harshfield GA, Alpert BS, Willey ES, Somes GW, Murphy JK, Dupaul LM: Race and gender influence ambulatory blood pressure patterns of adolescents. Hypertension 1989; 14: 598-603.

42. Agyemang C, Bhopal R, Bruijnzeels M, Redekop WK: Does nocturnal blood pressure fall in people of African and South Asian descent differ from that in European white populations? A systematic review and meta-analysis. $J$ Hypertens 200; 23: 913-920.

43. Fukuda M, Munemura M, Usami T, et al: Nocturnal blood pressure is elevated with natriuresis and proteinuria as renal function deteriorates in nephropathy. Kidney Int 2004; 65: 621-625.

44. Kimura G: Sodium, kidney, and circadian rhythm of blood pressure. Clin Exp Nephrol 2001; 5: 13-18.

45. O'Brien E, Sheridan J, O'Malley K: Dippers and non-dippers. Lancet 1988; 2: 397.

46. Verdecchia P, Schillaci G, Guerrieri M, et al: Circadian 
blood pressure changes and left ventricular hypertrophy in essential hypertension. Circulation 1990; 81: 528-536.

47. Shimada K, Kawamoto A, Matsubayashi K, Ozawa T: Silent cerebrovascular disease in the elderly. Correlation with ambulatory pressure. Hypertension 1990; 16: 692-699.

48. Verdecchia P, Porcellati C, Schillaci G, et al: Ambulatory blood pressure. An independent predictor of prognosis in essential hypertension. Hypertension 1994; 24: 793-801.

49. Pickering TG: The clinical significance of diurnal blood pressure variations. Dippers and nondippers. Circulation 1990; 81: 700-702.

50. Staessen JA, Thijs L, Fagard R, et al, Systolic Hypertension in Europe Trial Investigators: Predicting cardiovascular risk using conventional vs ambulatory blood pressure in older patients with systolic hypertension. JAMA 1999; 282: 539546.

51. Mancia G, Facchetti R, Bombelli M, et al: Relationship of office, home, and ambulatory blood pressure to blood glucose and lipid variables in the PAMELA population. Hypertension 2005; 45: 1072-1077.

52. Dolan E, Stanton A, Thijs L, et al: Superiority of ambulatory over clinic blood pressure measurement in predicting mortality: the Dublin outcome study. Hypertension 2005; 46: $156-161$.

53. Mann JF, Gerstein HC, Pogue J, Bosch J, Yusuf S: Renal insufficiency as a predictor of cardiovascular outcomes and the impact of ramipril: the HOPE randomized trial. Ann Intern Med 2001; 134: 629-636.

54. K/DOQI clinical practice guidelines for chronic kidney disease: evaluation, classification, and stratification. Am J Kidney Dis 2002; 39 (2 Suppl 1): S1-S266.

55. Sarnak MJ, Levey AS, Schoolwerth AC, et al: Kidney disease as a risk factor for development of cardiovascular disease: a statement from the American Heart Association Councils on Kidney in Cardiovascular Disease, High Blood Pressure Research, Clinical Cardiology, and Epidemiology and Prevention. Circulation 2003; 108: 2154-2169.

56. Ritz E, McClellan WM: Overview: increased cardiovascular risk in patients with minor renal dysfunction: an emerging issue with far-reaching consequences. $J$ Am Soc Nephrol 2004; 15: 513-516.

57. Go AS, Chertow GM, Fan D, McCulloch CE, Hsu CY: Chronic kidney disease and the risks of death, cardiovascular events, and hospitalization. $N$ Engl J Med 2004; 351: 1296-1305.

58. Adler AI, Stevens RJ, Manley SE, Bilous RW, Cull CA, Holman RR: Development and progression of nephropathy in type 2 diabetes: the United Kingdom Prospective Diabetes Study (UKPDS 64). Kidney Int 2003; 63: 225-232.

59. Goto N, Uchida K, Morozumi K, et al: Circadian blood pressure rhythm is disturbed by nephrectomy. Hypertens Res 2005; 28: 301-306.

60. Kimura G: Glomerular function reserve and sodium sensitivity. Clin Exp Nephrol 2005; 9: 102-113.

61. Morimoto A, Uzu T, Fujii T, et al: Sodium sensitivity and cardiovascular events in patients with essential hypertension. Lancet 1997; 350: 1734-1737.

62. Kimura G: Sodium sensitivity of blood pressure: a new prognostic factor in hypertension. Nephron 1999; 83: 97 105.
63. Weinberger MH, Fineberg NS, Fineberg SE, Weinberger M: Salt sensitivity, pulse pressure, and death in normal and hypertensive humans. Hypertension 2001; 37 (2 Part 2): 429-432.

64. Law MR, Wald NJ, Morris JK, Jordan RE: Value of low dose combination treatment with blood pressure lowering drugs: analysis of 354 randomised trials. BMJ 2003; 326: 1427.

65. Siscovick DS, Raghunathan TE, Psaty BM, et al: Diuretic therapy for hypertension and the risk of primary cardiac arrest. N Engl J Med 1994; 330: 1852-1857.

66. Franse LV, Pahor M, Di Bari M, Somes GW, Cushman WC, Applegate WB: Hypokalemia associated with diuretic use and cardiovascular events in the Systolic Hypertension in the Elderly Program. Hypertension 2000; 35: 1025-1030.

67. Verdecchia P, Reboldi G, Angeli F, et al: Adverse prognostic significance of new diabetes in treated hypertensive subjects. Hypertension 2004; 43: 963-969.

68. Kostis JB, Wilson AC, Freudenberger RS, Cosgrove NM, Pressel SL, Davis BR: Long-term effect of diuretic-based therapy on fatal outcomes in subjects with isolated systolic hypertension with and without diabetes. Am J Cardiol 2005; 95: 29-35.

69. Inaba M, Noguchi Y, Yamamoto T, et al: Effects of a low dose of indapamide, a diuretic, given daily or every-otherday on blood pressure and metabolic parameters. Hypertens Res 2004; 27: 141-145.

70. Doulton TW, He FJ, MacGregor GA: Systematic review of combined angiotensin-converting enzyme inhibition and angiotensin receptor blockade in hypertension. Hypertension 2005 ; 45: 880-886.

71. Dahlof B, Devereux RB, Kjeldsen SE, et al: Cardiovascular morbidity and mortality in the Losartan Intervention for Endpoint Reduction in Hypertension Study (LIFE): a randomised trial against atenolol. Lancet 2002; 359: 995-1003.

72. Lindholm LH, Ibsen H, Dahlof B, et al: Cardiovascular morbidity and mortality in patients with diabetes in the Losartan Intervention for Endpoint Reduction in Hypertension Study (LIFE): a randomised trial against atenolol. Lancet 2002; 359: 1004-1010.

73. Dahlof B, Devereux RB, Kjeldsen SE: Diuretics in the LIFE study. Lancet 2004; 364: 413-414.

74. Dahlof B, Sever PS, Poulter NR, et al: Prevention of cardiovascular events with an antihypertensive regimen of amlodipine adding perindopril as required versus atenolol adding bendroflumethiazide as required, in the Anglo-Scandinavian Cardiac Outcomes Trial-Blood Pressure Lowering Arm (ASCOT-BPLA): a multicentre randomised controlled trial. Lancet 2005; 366: 895-906.

75. Poulter NR, Wedel H, Dahlof B, et al: Role of blood pressure and other variables in the differential cardiovascular event rates noted in the Anglo-Scandinavian Cardiac Outcomes Trial-Blood Pressure Lowering Arm (ASCOTBPLA). Lancet 2005; 366: 907-913.

76. Epstein M, Messerli FM: Combination therapy in the management of hypertension, in Messerli FM (ed): The ABCs of Antihypertensive Therapy. New York, Authors' Publishing House, 2000; pp 204-221.

77. Kaplan NM: Clinical Hypertension. Philadelphia, Lippincott, 2002. 